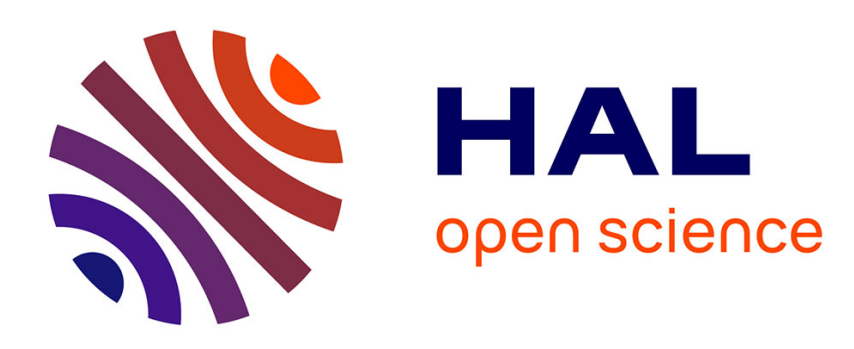

\title{
Overview of the application of anaerobic treatment to chemical and petrochemical wastewaters
}

Hervé Macarie

\section{To cite this version:}

Hervé Macarie. Overview of the application of anaerobic treatment to chemical and petrochemical wastewaters. Water Science and Technology, 2000, 42 (5-6), pp.201-213. 10.2166/wst.2000.0515 . ird-02089618v2

\section{HAL Id: ird-02089618 \\ https://hal.ird.fr/ird-02089618v2}

Submitted on 5 Apr 2019

HAL is a multi-disciplinary open access archive for the deposit and dissemination of scientific research documents, whether they are published or not. The documents may come from teaching and research institutions in France or abroad, or from public or private research centers.
L'archive ouverte pluridisciplinaire HAL, est destinée au dépôt et à la diffusion de documents scientifiques de niveau recherche, publiés ou non, émanant des établissements d'enseignement et de recherche français ou étrangers, des laboratoires publics ou privés. 


\title{
OVERVIEW ON THE APPLICATION OF ANAEROBIC TREATMENT TO CHEMICAL AND PETROCHEMICAL WASTEWATERS
}

\author{
Hervé Macarie \\ Institut de Recherche pour le Développement (IRD-France, ex-ORSTOM) \\ Cicerón 609, Col. Los Morales, 11530 México D. F., Mexico \\ Email:herve.macarie@ird.fr
}

\begin{abstract}
During the last 20 years, as a result of its low cost, anaerobic digestion has turned into a popular wastewater treatment technology. Today, with at least 1330 reactors constructed in the world, it is considered to have reached the technological maturity. Until recently however, it was used quite exclusively for the treatment of food industry effluents. It is only during the last 10 years that anaerobic digestion has started to be applied massively to the treatment of sewage and effluents from other industrial activities. During the 70's and 80's, the chemical and petrochemical industries were almost refractory to the introduction of anaerobic digestion. The situation has reversed since 1990 and at least 80 full-scale anaerobic plants are nowadays treating this type of waste in the world. Nevertheless, a great amount of promotion is still required before anaerobic digestion can be considered as an accepted technology by this industry. The paper presents the actual situation of anaerobic treatment at fullscale in this industrial sector as well as recent development at lab-scale and discuss some important concepts to consider before the implementation of an anaerobic treatment. Particularly a table is given with the main characteristics of 62 of the 80 full-scale plants identified to date. The probable reasons for the slow initial development of anaerobic treatment are also discussed and it is shown that anaerobic digestion has been the solution to treatment problems for which aerobic systems were inefficient.
\end{abstract}

\section{KEYWORDS}

Anaerobic treatment; chemical; petrochemical; wastewater.

\section{INTRODUCTION}

During the last 20 years, anaerobic digestion (AD), a biological process in which organic matter is converted to $\mathrm{CH}_{4}$ and $\mathrm{CO}_{2}$, has grown more and more into an attractive technology for wastewater treatment due to its low cost compared to the other technologies available: physicochemical and aerobic biological treatments. Its apparent initial drawbacks (very slow growth rate of the biomass, susceptibility to toxic compounds...), translated in huge reactor volumes and operation upsets, were overcame by the development of a new generation of reactors. In these reactors, the problem of slow growth rate was turned by capturing the biomass in the form of biofilms on static (Upflow Anaerobic Filters: UAF, Downflow Stationary Fixed Film reactors: DSFF) or moving (Fluidised Bed reactors: FB) supports but also by selecting well settling flocculating biomass (Anaerobic Contact: AC, Upflow Anaerobic Sludge Blanket reactors: UASB, Expanded Granular Sludge Bed reactors: EGSB, Internal Circulation reactors: IC). With such modifications, the sludge retention time in these reactors became independent of the hydraulic retention time (HRT) allowing the application of short HRTs ( $6 \mathrm{~h}$ to 1 week) and correspondingly the application of high organic loading rates (4 to $40 \mathrm{~kg} \mathrm{COD} / \mathrm{m}^{3}$ reactor $/$ day). This resulted in much smaller reactors but also in a much more stable operation than before.

Nowadays, with at least 1330 low and high rates reactors constructed in the world (Table 1) anaerobic digestion is considered to have reached the technological maturity. Other inventory indicates, even, a number of anaerobic plants in excess of 2000 (Totzke, 1999). Until today, however, anaerobic treatment has been applied quite exclusively ( $76 \%$ of all the reactors in operation) to the treatment of wastewaters from the food 
Table 1. Estimated number of the full scale reactors built in the world for treating several kinds of wastewaters $(*)$

\begin{tabular}{lllllllll}
\hline & \multicolumn{1}{c}{ Type of reactor (**) } \\
Type of Wastewater & $\begin{array}{l}\text { Low rate } \\
\text { \& CSTR }\end{array}$ & AC & UAF & DSFF Hybrid UASB & EGSB & IC & FB & Total \\
number
\end{tabular}

\section{Food and related industries}

Brewery and malt
Distillery \& ethanol
Food and fruit processing
Sugar production
Soft drinks and tea beverage
Potato processing
Starch production
Dairy and cheese
Yeast production
Candy/Confectionery/Chewing gum
Slaughterhouse and meat rendering
Fruit Juice
Cannery
Citric acid production
Wine processing
Coffee processing
Vegetable processing
Fish \& Sea food processing
Ice cream production
Jam production
Bakery
Pectin production
Fermentation
Tobacco manufacture
Chocolate processing
Store garbage
Guar gum manufacture
Cooking oil production

\section{Non Food Industries}

\begin{tabular}{|c|c|c|c|c|c|c|c|c|c|c|}
\hline Pulp and paper & 1 & 14 & 4 & - & 2 & 75 & - & 7 & 3 & 106 \\
\hline Petrochemical and chemical & 3 & 4 & 17 & 12 & 11 & 23 & 9 & - & 1 & 80 \\
\hline Leachates & - & - & 1 & 1 & 1 & 17 & - & - & - & 20 \\
\hline Pharmacy & 4 & 2 & 2 & 1 & - & 5 & 3 & - & - & 17 \\
\hline Pig, cow manure \& poultry & 5 & 3 & 3 & 2 & - & 1 & - & - & - & 14 \\
\hline Natural rubber & - & - & 3 & - & - & 3 & - & - & - & 6 \\
\hline Sludge liquor & - & - & 1 & - & 1 & 2 & - & - & 1 & 5 \\
\hline Textile & - & - & - & - & - & 3 & - & - & - & 3 \\
\hline Tannery & - & - & - & - & - & 2 & - & - & - & 2 \\
\hline Flue gas desulfurization & - & - & - & - & - & - & 1 & - & - & 1 \\
\hline Electronic components manufacture & - & - & - & - & - & 1 & - & - & - & 1 \\
\hline ewage & - & - & 2 & - & 1 & 58 & - & - & - & 61 \\
\hline Number of reactors per type & 83 & 138 & 91 & 54 & 51 & 772 & 54 & 61 & 19 & 1330 \\
\hline
\end{tabular}


and related industries (bakery, brewery, cannery, dairy, distillery, fish and potatoes processing, malting, candy, citric acid, coffee, cheese, chocolate, enzyme, fruit juice, jam, soft drink, starch, sugar, wine and yeast productions) and it is only recently (over the last 10 years) that it started to be applied massively to sewage (4.6\% of all the digesters in operation) as well as other industrial sectors such as the pulp and paper (8\% of the operating digesters).

What happened during this time in the chemical and petrochemical industries?

\section{DEVELOPMENT OF AD AMONG (PETRO)CHEMICAL INDUSTRIES}

The first studies about the anaerobic treatment of this type of wastewaters started at the beginning of the 70's. In 1973, for instance, Hovious et al. demonstrated at pilot scale the possibility to use an anaerobic lagoon as an efficient pre-treatment for petrochemical effluents. Few years later, Chou et al. (1978) published a list of 41 organic compounds potentially present in petrochemical and chemical industry effluents that they found to be biodegradable by methanogenic fermentation. Despite these early works, it is only in 1981 that the 2 first high rate digesters treating chemical waste were built by the Celanese company in USA (Table 2). Three more years passed before a third reactor was built and until 1989 as far investigated, only 16 full-scale reactors were in operation on that type of waste in the world (Table 2). From 1990 to date, the rate of construction of digesters for that industrial sector increased from 2.1 reactors/year for the past decade to 4.6 and presently, at least 80 digesters (6\% of all the digesters) are treating chemical waste in the world (Table 1). Detailed information is given about 62 of them in Table 2. No data were available for the others ( 7 built by Biothane, 5 by Amoco Co., 1 by Biotim and 1 by Enviroasia).

The initial slow development of anaerobic digestion in that sector was probably the result of an "a priori" (both from the companies dedicated to the design of anaerobic systems than the chemical and petrochemical industries), postulating that anaerobes, particularly methanogens, contrarily to aerobes, were too sensible to deal with this type of wastewaters supposed to be highly toxic. In fact, a comparative study of tolerance to toxicity between aerobes and anaerobes has shown that such assumption is not justified (Blum and Speece, 1991). In some cases, anaerobic digestion has even appeared to be the key to the success of the degradation of some molecules. In 1981, for instance, the activated sludge treatment system of the Celanese company at Bishop, Texas, USA, was not able to treat 2 particular effluents. One of them, although composed of readily biodegradable molecules, had a concentration of heavy metals $(5-500 \mathrm{mg} / \mathrm{L})$ toxic for the aerobic bacteria, while the second contained polyols (pentaerythritol, trimethylolpropane) refractory to aerobic degradation. The implementation of an anaerobic reactor previous to the aerobic plant, by precipitating the heavy metals under the form of non toxic metallic sulfides, allowed to degrade the first effluent directly within the anaerobic stage and by modifying the chemical structure of the polyols, transformed them into compounds biodegradables in the aerobic post-treatment unit (Harvey and Rubiano, 1983).

\section{Nature of the wastewaters presently treated at full scale or which could be treated.}

As indicated in Table 2, the spectrum of wastewaters already treated at full scale results from a large number of very different industrial activities. Nevertheless, on the whole, the first anaerobic digestion installations were set up on relatively simple wastewaters, composed mostly of volatile fatty acids (reactors $2,4,5,6,7$, $10,12)$, methanol $(4,10)$ and glycols $(11)$ also found in more classical effluents for AD. If we except the case of Shell in 1986 which contained a high concentration of benzoic acid (reactor 8) and that of Celanese in 1981 (reactor 1), it is only in 1989, with the installation by Amoco of a digester on PTA (Purified Terephthalic Acid) wastewater, that a really unusual effluent containing aromatic compounds from the benzenic series was treated (reactor 14). Moreover, It should be noted that it is one of the few effluents, which has given rise to the construction of a series of anaerobic plants, 14 until now. In fact, in its case, anaerobic digestion seems, even, to be on the way to become the conventional form of treatment. The other activities which resulted in the construction of more than one reactor are the production of DMT (dimethylterephthalate, 4 plants), PET (polyethylene terephthalate, 6 plants) and aspartame (2 plants). All the other reactors correspond however to single experiences. It is evident, that the chemical and petrochemical effluents treatable by anaerobic digestion are not limited to those presented in Table 2. Already, several others have been successfully treated anaerobically at pilot or lab scale (Table 3). A great number of molecules susceptible to be produced by this type of industrial activity and then to be present in the wastewaters are also known to be biodegradable by methanogenic fermentation (Table 4). Then it should be expected to see in the future, the application of anaerobic digestion to a growing number of chemical wastewaters. 
Table 2. Full scale anaerobic digesters treating chemical and petrochemical wastewaters in the world $(*)$

\begin{tabular}{|c|c|c|c|c|c|c|c|c|c|}
\hline $\begin{array}{l}\text { Reactor } \\
\text { number }\end{array}$ & $\begin{array}{c}\text { Year } \\
\text { of } \\
\text { construction }\end{array}$ & $\begin{array}{l}\text { Company } \\
\text { and } \\
\text { location }\end{array}$ & $\begin{array}{l}\text { Industrial production } \\
\text { generating the } \\
\text { wastewater }\end{array}$ & $\begin{array}{l}\text { Type } \\
\text { of } \\
\text { reactor }\end{array}$ & $\begin{array}{c}\text { Reactor } \\
\text { volume } \\
\mathrm{m}^{3}\end{array}$ & $\begin{array}{l}\text { Water } \\
\text { COD } \\
\mathrm{g} \mathrm{O}_{2} / \mathrm{L}\end{array}$ & $\begin{array}{l}\text { Organic } \\
\text { Load } \\
\mathrm{kg} \mathrm{COD} / \mathrm{m}^{3} \cdot \mathrm{d}\end{array}$ & $\begin{array}{c}\text { COD } \\
\text { removal } \\
\%\end{array}$ & $\begin{array}{c}\text { Constructor } \\
/ \\
\text { references } \\
(* *)\end{array}$ \\
\hline 1 & 1981 & $\begin{array}{l}\text { Celanese } \\
\text { Bishop, TX, USA }\end{array}$ & $\begin{array}{l}\text { Acetic acid, formaldehyde, } \\
\text { methanol, polyols, polyesters }\end{array}$ & UAF & 5682 & 7.12 & 3.6 & 81 & Badger $^{1}$ \\
\hline 2 & 1981 & $\begin{array}{l}\text { Celanese } \\
\text { Pampa, TX } \\
\text { USA }\end{array}$ & $\begin{array}{l}\text { Acetic, propionic, butyric } \\
\text { and anhydride acetic acids } \\
\text { Ketones, ethylacetate, } \\
\text { Acrylic esters }\end{array}$ & UAF & 5229 & 13.3 & 10.4 & 80 & Badger $^{2}$ \\
\hline 3 & 1984 & $\begin{array}{l}\text { Hercules, Alizay } \\
\text { France }\end{array}$ & Carboxymethylcellulose & - & 3000 & - & 1.7 & 87 & $\begin{array}{l}\text { Biome- } \\
\text { chanics }\end{array}$ \\
\hline 4 & 1985 & $\begin{array}{l}\text { Monsanto Corp } \\
\text { Augusta, GA, USA }\end{array}$ & Aspartame & UAF & $\begin{array}{l}2 \times 1900 \\
\text { in series }\end{array}$ & 12 & $\begin{array}{l}3-4 \\
6-8\end{array}$ & $\begin{array}{l}90-95 \\
85-90\end{array}$ & $-2,4$ \\
\hline 5 & 1986 & $\begin{array}{l}\text { DSM Chemicals } \\
\text { Rotterdam, Netherlands }\end{array}$ & Phenol & UASB & 1280 & 30.5 & $9-12$ & 95 & Biothane $^{5}$ \\
\hline 6 & 1986 & $\begin{array}{l}\text { Hoechst } \\
\text { Lillebonne, France }\end{array}$ & $\begin{array}{l}\text { Acetaldehyde } \\
\text { Glyoxylic acid }\end{array}$ & $\mathrm{AC}$ & 3000 & 43 & 5.5 & 98 & Degrémont $^{6}$ \\
\hline 7 & 1987 & $\begin{array}{l}\text { Hoechst } \\
\text { Cuise-Lamotte } \\
\text { France }\end{array}$ & $\begin{array}{l}\text { Glyoxylic acid and glyoxane } \\
\text { Paratertiobutylbenzoic acid } \\
\text { Tienylacetic acid, hydantoine }\end{array}$ & DSFF & 2150 & $45-50$ & 7.4 & 90 & Proserpol $^{6,7}$ \\
\hline 8 & 1987 & $\begin{array}{l}\text { Shell Chemie } \\
\text { Moerdijk, Netherlands }\end{array}$ & $\begin{array}{l}\text { Methylstyrene and } \\
\text { Propene oxides }\end{array}$ & UASB & 1430 & $20-45$ & $10-20$ & $80-95$ & Biothane $^{8}$ \\
\hline 9 & 1987 & $\begin{array}{l}\text { Toban Dyeing, } \\
\text { Hyogo, Japan }\end{array}$ & Dyeing wastewater & UAF & - & - & - & - & $\begin{array}{l}\text { Shinko } \\
\text { Pantec }\end{array}$ \\
\hline 10 & 1988 & JGC, Kanagawa, Japan & Synthetic resin & UAF & 260 & 10.5 & 8 & 75 & $\begin{array}{l}\text { Shinko } \\
\text { Pantec }\end{array}$ \\
\hline 11 & 1988 & $\begin{array}{l}\text { Orient Chemical } \\
\text { Osaka, Japan }\end{array}$ & Dyes & UAF & 320 & 7 & 7 & 80 & $\begin{array}{l}\text { Shinko } \\
\text { Pantec }\end{array}$ \\
\hline 12 & 1988 & $\begin{array}{l}\text { Shin Etsu chemical } \\
\text { Nigata, Japan }\end{array}$ & Synthetic cellulose & UAF & 2350 & 12.6 & 8 & 65 & $\begin{array}{l}\text { Shinko } \\
\text { Pantec }\end{array}$ \\
\hline 13 & 1988 & $\begin{array}{l}\text { GLI corp. } \\
\text { Newport, TN, USA }\end{array}$ & $\begin{array}{l}\text { Artificial sweetener } \\
\text { (sucralose) }\end{array}$ & BVF & 26500 & 8.3 & 0.83 & 75 & ADI \\
\hline 14 & 1989 & $\begin{array}{l}\text { Capco Co. } \\
\text { Taiwan }\end{array}$ & Purified terephthalic acid & DSFF & $2 \times 5000$ & 10 & $3-4$ & $\begin{array}{c}85 \\
\text { (TOC) }\end{array}$ & Amoco $^{9}$ \\
\hline 15 & 1989 & $\begin{array}{l}\text { Cheil Synthetic textiles } \\
\text { Gumi, Korea }\end{array}$ & Polyester resins & UAF & - & - & - & - & Badger \\
\hline 16 & 1989 & $\begin{array}{l}\text { Shell oil Co., } \\
\text { Deer Park, TX, USA }\end{array}$ & Chemical processing & UAF & - & - & - & - & Badger \\
\hline 17 & 1990 & China & Purified terephthalic acid & Hybrid & $4 \times 3000$ & 9 & 6.3 & 80 & -10 \\
\hline 18 & 1990 & $\begin{array}{l}\text { Sam Nam, Korea } \\
\text { (+ extension 1994) }\end{array}$ & Purified terephthalic acid & $\begin{array}{l}\mathrm{AC} \\
\text { Hybrid }\end{array}$ & $\begin{array}{l}2000 \\
2 \times 1100 \\
\text { In series }\end{array}$ & $\begin{array}{l}12.6 \\
12.4\end{array}$ & $\begin{array}{c}4.35 \\
9\end{array}$ & $\begin{array}{l}75 \\
90\end{array}$ & $\begin{array}{l}\text { Purac }^{11} \\
\text { ADI }^{11,12}\end{array}$ \\
\hline 19 & 1991 & Tuntex, Taiwan. & Purified terephthalic acid & UASB & 7000 & $6-13$ & 10 & 55 & Grontmij $^{13}$ \\
\hline 20 & 1992 & Nigata, Japan & Carboxymethylcellulose & UAF & 1210 & 7.8 & 7 & 75 & $\begin{array}{l}\text { Shinko } \\
\text { Pantec }\end{array}$ \\
\hline 21 & 1992 & Okayama, Japan & Ligth oil from asphalt & UAF & 1025 & 8 & 6 & 55 & $\begin{array}{l}\text { Shinko } \\
\text { Pantec }\end{array}$ \\
\hline 22 & 1992 & $\begin{array}{l}\text { Amoco Co } \\
\text { Joliet, IL, USA }\end{array}$ & $\begin{array}{l}\text { Purified terephthalic } \\
\text { and isophthalic acids }\end{array}$ & DSFF & 8200 & - & 3.5 & $\begin{array}{c}85 \\
\text { (TOC) }\end{array}$ & Amoco \\
\hline 23 & 1992 & $\begin{array}{l}\text { Mossref, Mossel Bay } \\
\text { South Africa }\end{array}$ & Synthetic fuels & DSFF & $3 \times 5000$ & 14.2 & 8.5 & 93 & Proserpol $^{14}$ \\
\hline 24 & 1992 & Unichema, Taiwan & Oleochemicals (glycerine) & DSFF & 400 & 4.4 & 4.8 & 70 & Proserpol \\
\hline 25 & 1992 & $\begin{array}{l}\text { Samyang Co } \\
\text { Seoul, Korea }\end{array}$ & Plastics & UASB & 840 & 15 & 9.9 & - & Biothane \\
\hline 26 & 1992 & $\begin{array}{l}\text { Bombay Dyeing } \\
\text { Patalganga, India }\end{array}$ & DMT & UASB & 1500 & 20 & 8 & 70 & Paques \\
\hline
\end{tabular}


Table 2. Full scale anaerobic digesters treating chemical and petrochemical wastewaters in the world (continuation)

\begin{tabular}{|c|c|c|c|c|c|c|c|c|c|}
\hline $\begin{array}{l}\text { Reactor } \\
\text { number }\end{array}$ & $\begin{array}{c}\text { Year } \\
\text { of } \\
\text { construction }\end{array}$ & $\begin{array}{l}\text { Company } \\
\text { and } \\
\text { location }\end{array}$ & $\begin{array}{l}\text { Industrial production } \\
\text { generating the } \\
\text { wastewater }\end{array}$ & $\begin{array}{l}\text { Type } \\
\text { of } \\
\text { reactor }\end{array}$ & $\begin{array}{c}\text { Reactor } \\
\text { volume } \\
\mathrm{m}^{3}\end{array}$ & $\begin{array}{l}\text { Water } \\
\text { COD } \\
\mathrm{g} \mathrm{O}_{2} / \mathrm{L}\end{array}$ & $\begin{array}{l}\text { Organic } \\
\text { Load } \\
\mathrm{kg} \mathrm{COD} / \mathrm{m}^{3} \cdot \mathrm{d}\end{array}$ & $\begin{array}{c}\text { COD } \\
\text { removal } \\
\%\end{array}$ & $\begin{array}{c}\text { Constructor } \\
/ / \\
\text { references } \\
(* *)\end{array}$ \\
\hline 27 & 1992 & $\begin{array}{l}\text { Dae Han } \\
\text { Ulsan, Korea }\end{array}$ & Diethylene glycol & UASB & $2 \times 82$ & 3.6 & 7.5 & - & Biothane \\
\hline 28 & 1992 & $\begin{array}{l}\text { Tonen Chemical } \\
\text { Kawasaki, Japan }\end{array}$ & Maleic acid & UASB & 100 & 13.6 & 17.8 & 90 & Paques \\
\hline 29 & 1992 & $\begin{array}{l}\text { Nutrasweet Co. } \\
\text { Univ. Park, IL, USA }\end{array}$ & Aspartame & UASB & $2 \times 600$ & 22 & 7.8 & - & Biothane \\
\hline 30 & 1992 & $\begin{array}{l}\text { Caldic Europoort } \\
\text { Netherlands }\end{array}$ & Formaldehyde & EGSB & 275 & 40 & 17 & 98 & Biothane $^{15}$ \\
\hline 31 & 1992 & $\begin{array}{l}\text { Northwest Pipeline Co. } \\
\text { Opal, Wyoming, USA }\end{array}$ & Natural Gas processing & UAF & $\begin{array}{l}2 \times 108 \\
\text { in series }\end{array}$ & 13.4 & 8 & $90-95$ & $\begin{array}{l}\text { EnviroSys- } \\
\text { tems Inc }\end{array}$ \\
\hline 32 & 1993 & $\begin{array}{l}\text { Amoco Co. } \\
\text { Geel, Belgium }\end{array}$ & $\begin{array}{l}\text { Purified terephthalic \& } \\
\text { Isophthalic acids }\end{array}$ & DSFF & 15200 & 16.7 & 3.7 & $\begin{array}{c}80 \\
\text { (TOC) }\end{array}$ & Amoco $^{17}$ \\
\hline 33 & 1993 & $\begin{array}{l}\text { Exxon Co, Santa } \\
\text { Barbara, Ca, USA }\end{array}$ & Oil well produced water & UAF & - & - & - & - & Badger \\
\hline 34 & 1993 & Petrocel, Mexico & DMT & UASB & $2 \times 2400$ & 18.5 & 7.5 & 95 & Biothane \\
\hline 35 & 1993 & $\begin{array}{l}\text { Hoechst Celanese } \\
\text { Calisbury, NC, USA }\end{array}$ & Polyester resin & UAF & - & - & - & - & Badger \\
\hline 36 & 1994 & $\begin{array}{l}\text { Robertet } \\
\text { Grasse, France }\end{array}$ & Perfumes & FB & 92 & 8.4 & 27.7 & 94 & Degrémont \\
\hline 37 & $\begin{array}{c}1994 \\
\text { (ext. 1997) }\end{array}$ & $\begin{array}{l}\text { Reliance Industries } \\
\text { Hazira, India }\end{array}$ & Purified terephthalic acid & Hybrid & $\begin{array}{l}2 \times 3076 \\
2 \times 4190\end{array}$ & $\begin{array}{c}8.3 \\
6.28\end{array}$ & $\begin{array}{l}4.8 \\
5.3\end{array}$ & $\begin{array}{l}66 \\
70\end{array}$ & $\mathrm{ADI}^{11}$ \\
\hline 38 & 1994 & $\begin{array}{l}\text { Akso-Nobel } \\
\text { Emmen, Netherlands }\end{array}$ & Aramid fibers & UASB & 1400 & 0.65 & 3.8 & 60 & Paques \\
\hline 39 & 1994 & $\begin{array}{l}\text { ATV petrochemicals } \\
\text { Mathura, India }\end{array}$ & Purified terephthalic acid & $\begin{array}{l}\text { UASB+UAF } \\
\text { in series }\end{array}$ & $\begin{array}{c}1330 \\
\text { (UASB) }\end{array}$ & 12 & $10-12$ & $>60$ & Paques \\
\hline 40 & 1994 & Aussapol, Italy & PET & Hybrid & 750 & 12 & 1.6 & 77.5 & Biotim \\
\hline 41 & 1994 & Tuntex, Thailand & Purified terephthalic acid & UASB & $3 \times 3000$ & 10 & 6 & - & Hepe ${ }^{18}$ \\
\hline 42 & 1995 & $\begin{array}{l}\text { Tae Kwang } \\
\text { Korea }\end{array}$ & Polyesters & Hybrid & 500 & 20 & 10 & 80 & ADI \\
\hline 43 & 1995 & TNT Thailand & Nylon and PET fibers & BVF & 2700 & 9 & 1 & 80 & ADI \\
\hline 44 & 1995 & $\begin{array}{l}\text { Castagna Unilevel } \\
\text { Italy }\end{array}$ & $\begin{array}{l}\text { Ethyl acetate recovery from } \\
\text { rotagravure printing }\end{array}$ & UASB & 16 & 5 & 4.7 & 98 & Biothane \\
\hline 45 & 1995 & $\begin{array}{l}\text { DuPont de Nemours } \\
\text { Dordrecht, Netherlands }\end{array}$ & Thermoplastics & EGSB & 550 & 7.5 & 10 & 90 & Biothane ${ }^{19}$ \\
\hline 46 & 1996 & $\begin{array}{l}\text { SBI - Sanofi } \\
\text { Grasse, France }\end{array}$ & Perfumes & $\mathrm{AC}$ & 900 & - & 4.4 & 90 & OTVKruger $^{3}$ \\
\hline 47 & 1996 & $\begin{array}{l}\text { BKC } \\
\text { Indonesia }\end{array}$ & Purified terephthalic acid & $\mathrm{AC}$ & 4000 & $6-13$ & $1.7-2.3$ & $>80$ & Purac \\
\hline 48 & 1996 & $\begin{array}{l}\text { Eastman Chemical } \\
\text { Argentina }\end{array}$ & PET & UASB & 144 & 12 & 12 & $90-95$ & Biothane \\
\hline 49 & 1996 & $\begin{array}{l}\text { Volos PET Industry S.A., } \\
\text { Greece }\end{array}$ & PET & EGSB & 250 & 25 & 18 & 90 & Biothane \\
\hline 50 & 1996 & $\begin{array}{l}\text { Technoparco } \\
\text { Valbasento, Italy }\end{array}$ & Epichlorohydrin & UAF & 110 & $14-16$ & 8 & 83 & Eniricerche $^{20}$ \\
\hline 51 & 1996 & $\begin{array}{l}\text { SK Chemicals } \\
\text { Korea }\end{array}$ & $\begin{array}{l}\text { Polyesters and } \\
\text { Purified terephthalic acid }\end{array}$ & hybrid & 800 & 15.6 & 9 & 80 & ADI \\
\hline 52 & 1996 & $\begin{array}{l}\text { Garware Chemicals } \\
\text { Aurangabad, India }\end{array}$ & DMT and films & UASB & 1088 & - & 7 & - & Paques \\
\hline 53 & 1996 & $\begin{array}{l}\text { Rhône Poulenc } \\
\text { Chalampé, France }\end{array}$ & Nylon & UASB & 990 & 16 & 8 & 80 & Paques $^{21}$ \\
\hline
\end{tabular}


Table 2. Full scale anaerobic digesters treating chemical and petrochemical wastewaters in the world (continuation)

\begin{tabular}{|c|c|c|c|c|c|c|c|c|c|}
\hline $\begin{array}{l}\text { Reactor } \\
\text { number }\end{array}$ & $\begin{array}{c}\text { Year } \\
\text { of } \\
\text { construction }\end{array}$ & $\begin{array}{l}\text { Company } \\
\text { and } \\
\text { location }\end{array}$ & $\begin{array}{l}\text { Industrial production } \\
\text { generating the } \\
\text { wastewater }\end{array}$ & $\begin{array}{l}\text { Type } \\
\text { of } \\
\text { reactor }\end{array}$ & $\begin{array}{c}\text { Reactor } \\
\text { volume } \\
\mathrm{m}^{3}\end{array}$ & $\begin{array}{l}\text { Water } \\
\text { COD } \\
\mathrm{g} \mathrm{O}_{2} / \mathrm{L}\end{array}$ & $\begin{array}{l}\text { Organic } \\
\text { Load } \\
\mathrm{kg} \mathrm{COD} / \mathrm{m}^{3} . \mathrm{d}\end{array}$ & $\begin{array}{c}\text { COD } \\
\text { removal } \\
\%\end{array}$ & $\begin{array}{c}\text { Constructor } \\
/ \\
\text { references } \\
(* *)\end{array}$ \\
\hline 54 & 1997 & $\begin{array}{l}\text { Reliance Industries } \\
\text { Patalganga, India }\end{array}$ & Purified terephthalic acid & hybrid & $\begin{array}{c}800 \\
(* * *)\end{array}$ & 8 & 5.4 & 52 & $\mathrm{ADI}^{22}$ \\
\hline 55 & 1997 & $\begin{array}{l}\text { Catalana de polimers } \\
\text { Barcelona, Spain }\end{array}$ & PET & UASB & 635 & 30 & 10 & 90 & Arema $^{23}$ \\
\hline 56 & 1998 & $\begin{array}{l}\text { Dupont Far Eastern } \\
\text { Petroch. Ltd, Taiwan }\end{array}$ & Purified terephthalic acid & hybrid & $\begin{array}{l}1 \times 5000 \\
1 \times 4000\end{array}$ & 6.5 & 5.6 & 65 & ADI \\
\hline 57 & 1998 & $\begin{array}{l}\text { Eastman Chemical } \\
\text { Malaysia }\end{array}$ & Ethylene glycol & hybrid & $2 \times 33$ & 6.45 & 2.6 & 87 & ADI \\
\hline 58 & 1998 & $\begin{array}{l}\text { Kosa, Vlissingen } \\
\text { Netherlands }\end{array}$ & DMT & EGSB & 550 & 33.8 & 13.2 & - & Biothane \\
\hline 59 & 1998 & Sasa, Turkey & DMT \& PET & EGSB & $2 \times 1000$ & 6.5 & 13 & - & Biothane \\
\hline 60 & 1998 & $\begin{array}{l}\text { Toray Plastics Europe } \\
\text { France }\end{array}$ & PET & DSFF & 400 & 5 & 5 & $70-80$ & Proserpol \\
\hline 61 & 1998 & $\begin{array}{l}\text { Temex } \\
\text { Mexico }\end{array}$ & $\begin{array}{l}\text { Purified terephthalic acid } \\
\& \text { PET }\end{array}$ & $\begin{array}{l}\text { Upflow } \\
\text { pond }\end{array}$ & 20000 & $6-12$ & $2-3$ & $60-70$ & IBtech $^{24}$ \\
\hline 62 & 1999 & $\begin{array}{l}\text { Rotapas } \\
\text { Italy }\end{array}$ & $\begin{array}{l}\text { Solvents recovery from a } \\
\text { print shop }\end{array}$ & UASB & 50 & 8 & 8 & - & Biothane \\
\hline
\end{tabular}

(*) The abbreviations are the same as in Table $1, \mathrm{BVF}=$ Bulk Volume Fermenter, TOC $=$ Total Organic Carbon, DMT $=$ Dimethylterephthalate, PET $=$ Polyethylene terephthalate (**) The information comes from the same source as in Table 1 and from the following articles: Harvey and Rubiano (1983) ${ }^{1}$, Young $(1991)^{2}$, Feuillette $(1996)^{3}$, Young and Young $(1991)^{4}$, Borghans and van Driel (1988) ${ }^{5}$, Roy and Durand (1994) $)^{6}$, Henry and Varaldo (1988) ${ }^{7}$, Frankin et al. $(1994 b)^{8}$, Shelley $(1991)^{9}$, Macarie et al. $(1992)^{10}$, Page et al. $(1998)^{11}$, Young et al. $(2000)^{12}$, Pereboom et al. $(1994)^{13}$, Marx $(1994)^{14}$, Zoutberg and de Been $(1997)^{15}$, Ferrel and Young $(1993)^{16}$, Vanduffel $(1993)^{17}$, Kleerebezem $(1999)^{18}$, Constable and Kras $(1998)^{19}$, Anonym $(1996)^{20}$, Boulenger et al. $(2000)^{21}$, Page et al. $(1999)^{22}$, Fdz-Polanco et al. $(1999)^{23}$, Noyola et al. $(2000)^{24} .(* * *)$ Refurbished from an existing anaerobic FB reactor designed by Dorr-Oliver.

Table 3. Laboratory studies showing the possibility to apply anaerobic digestion to chemical effluents untreated by this way at full scale until now

\begin{tabular}{|c|c|c|c|c|c|}
\hline Type of wastewater $(*)$ & $\begin{array}{c}\text { Type of } \\
\text { reactor } \\
(* *)\end{array}$ & $\begin{array}{c}\text { Reactor } \\
\text { volume } \\
\text { L }\end{array}$ & $\begin{array}{c}\text { Wastewater } \\
\text { COD } \\
\mathrm{g} \mathrm{O}_{2} / \mathrm{L}\end{array}$ & $\begin{array}{l}\text { Organic loading } \\
\text { rate } \\
\mathrm{kg} \mathrm{DCO} / \mathrm{m}^{3} . \mathrm{d}\end{array}$ & $\begin{array}{c}\text { COD } \\
\text { removal } \\
\%\end{array}$ \\
\hline $\begin{array}{l}\text { Production of acrylic acid and related } \\
\text { esters }\end{array}$ & UAF & 5 & 19 & 2.6 & 97 \\
\hline $\begin{array}{l}\text { Synthetic wastewater containing } \\
\text { hydroquinone }\end{array}$ & UAF & 0.5 & $1-4$ & $3.2-60$ & $47-100$ \\
\hline Refinery sour water stripper bottoms ${ }^{3}$ & FB with $\mathrm{GAC}(* *)$ & - & 1.5 & $2-11$ & $63-91$ \\
\hline Furfural production ${ }^{4}$ & UAF & 9.5 & $10-16$ & 23 & 92 \\
\hline $\begin{array}{l}\text { Production of phenolic resins and phenol } \\
\text { molding compounds }\end{array}$ & FB with $\mathrm{GAC}(* *)$ & 30 & 39 & 5.6 & 98 \\
\hline $\begin{array}{l}\text { Platicizer production and effluent from a } \\
\text { resin distillation column }\end{array}$ & Hybrid & 1 & 17 & 12 & 58 \\
\hline $\begin{array}{l}\text { Synthetic effluent containing benzaldehyde } \\
\text { and saccharose }^{7}\end{array}$ & UASB & 2 & - & 4.8 & 84 \\
\hline $\begin{array}{l}\text { Production of styrene-divinylbenzene } \\
\text { polymeric resins }\end{array}$ & UASB & 6 & $8-10$ & 4.5 & 78 \\
\hline 2,4-dichlorophenoxyacetic acid production ${ }^{9}$ & FB with $\mathrm{GAC}(* *)$ & 710 & $2.5-6.3$ & $14-38$ & $85-90$ \\
\hline Styrene polymer synthesis plant ${ }^{10}$ & UASB & 4 & 2.2 & 4.3 & $>75$ \\
\hline
\end{tabular}

(*) Dohányos et al. (1988) ${ }^{1}$, Szewzyk and Schink $(1989)^{2}$, Gardner et al. $(1988)^{3}$, Wirtz and Dague (1993) ${ }^{4}$, Goeddertz et al. (1990) ${ }^{5}$, Nemer et al. $(1994)^{6}$, Todini and Hulshoff Pol $(1992)^{7}$, Dangcong et al. $(1994)^{8}$, Wilson et al. $(1997)^{9}$, Araya et al. $(1999)^{10}$. (**) GAC: Granular Activated Carbon 


\section{Necessity of pre-treatments}

Even if several chemical and petrochemical effluents cannot be methanised directly, because they contain organic compounds difficult to be treated anaerobically, toxic substances or an inadequate environment (e.g. high salinity), several pre-treatment systems are in fact available to solve these problems. The techniques of electrochemical (Pulgarin et al., 1994), chemical (Koyama et al., 1994) and photochemical (Yi et al., 1994) oxidation or else ozonation (Wang, 1990), by their ability to modify the structure of the molecules (cleavage of the aromatic nucleus and polymeric linear chains, introduction of oxygen within the structure) allow for instance to increase the biodegradability and decrease the toxicity of the effluents. On its side, the high salinity can be eliminated by a selective filtration through membranes permeables to organic substances but not mineral salts (Brookes and Livingston, 1994).

Without going to so sophisticated systems, which are moreover for most of them still at the experimental stage, a simple adjustment of $\mathrm{pH}$ may be the solution to toxicity problems. In this way, formaldehyde, which is strongly toxic to microorganisms because of its capacity to react with proteins and denature them, transforms spontaneously at high $\mathrm{pH}(11-12)$ and temperature $\left(100^{\circ} \mathrm{C}\right)$ in a mixture of sugars, methanol and formic acid. This technique has been applied successfully at pilot scale (UASB reactor of $6 \mathrm{~m}^{3}$ ) to detoxify the effluents from the production of DMT (formaldehyde concentration of 2-3 g/L) otherwise impossible to treat anaerobically unless applying a very high dilution (Bekker et al., 1983).

Other example of simple solutions is that selected for the effluents of PTA production. This type of wastewater, characterised by a $\mathrm{pH}$ of 4.5 and a temperature of $56^{\circ} \mathrm{C}$, contains a high concentration $(1-4 \mathrm{~g} / \mathrm{L})$ of terephthalic acid (1,4-benzenedicarboxylic acid, TA), which is poorly soluble in water $\left(19 \mathrm{mg} / \mathrm{L}\right.$ at $25^{\circ} \mathrm{C}$, $400 \mathrm{mg} / \mathrm{L}$ at $\left.100^{\circ} \mathrm{C}\right)$ and has a high density $\left(1.5 \mathrm{~g} / \mathrm{mL}\right.$ at $\left.25^{\circ} \mathrm{C}\right)$ (Macarie et al., 1992; Fajardo et al., 1997). These characteristics indicate that because of its particulate form, TA cannot be degraded significantly in high rates digesters operated with short hydraulic retention times. Its deposition in tanks and lines would also generate serious plugging problems of the reactor feeding tubes as well as displacement of the active biomass. Two solutions based on its physical properties are presently used at full scale. The first consists to withdraw it from the wastewater by primary settling without treating it in the anaerobic unit (reactor 17 in Table 2), and the second to transform it by a simple neutralisation (a minimum $\mathrm{pH}$ of 5.5 is necessary to avoid precipitation; Kleerebezem, 1999) in its much more soluble sodium salt $\left(140 \mathrm{~g} / \mathrm{L}\right.$ at $25^{\circ} \mathrm{C}$, Merck 1999/2000 catalogue of chemical products) and to treat it in the biological phase (reactors, 14, 22 and 32 in Table 2). A complete neutralisation with external alkaline compounds is however not necessary since the alkalinity produced within the anaerobic system can be valorised through effluent recirculation. The alkaline power of the recycled effluent may even be increased by stripping of its $\mathrm{CO}_{2}$ content (Ferguson et al., 1984). Such process, which reduces substantially the cost of neutralisation, has lead to the register of a patent (Ely and Olsen, 1989).

Two last examples of simple pre-treatments correspond to those applied at full scale in the case of Nylon wastewaters (Table 2, reactor 53) and effluents containing epichlorohydrin (1-chloro-2,3-epoxypropane), a solvent used in the manufacture of natural and synthetic resins, gums, cellulose esters and ethers as well as several other products (Table 2, reactor 50). Nylon wastewaters contain peroxides, which like oxygen are harmful to anaerobic bacteria. They can be easily eliminated by a combination of heat and catalytic treatment (Boulenger et al., 2000). Epichlorohydrin which is also toxic may be removed, from its side, through hot alkaline hydrolysis. The resulting effluent contains unfortunately an increased concentration of $\mathrm{NaCl}$ due to chloride liberation during the hydrolysis and must be further desalted with i.e. classical evaporation and crystallisation processes (Anonym, 1996).

The previous comments show that anaerobic digestion should not be eliminated straight away at the first problem and that the possibility to apply it to a chemical or petrochemical wastewater must be the result of a detailed evaluation.

\section{Type of reactors applied for the treatment of chemical and petrochemical effluents}

Despite the precursory work of Hovious et al. (1973), the technology of low rate reactors, such as anaerobic lagoons, seems to have found little echo in the (petro)chemical industry since only 3 reactors of this type have been constructed so far (Table 2 , reactor $13,43,61$ ). The situation is similar for the anaerobic contact digesters (Table 2 , reactors $6,18,46,47$ ). This may be due to the fact that chemical industries are familiar of « high tech » technologies, which means that they are probably more attracted by high rate processes. In this last category, all the types of digesters have been applied. The first realisations were based however on the technology of the upflow anaerobic filter and until 1989, they represented the majority $(56 \%, 9$ on 16$)$ of the installed reactors. 
Table 4. Non exhaustive list of organic compounds biodegradable by methanogenic fermentation and susceptible to be present in the effluents of chemical and petrochemical industries (after Macarie, 1992).

\begin{tabular}{|c|c|c|}
\hline Homocyclic aromatic compounds & Homocyclic aromatic compounds & Aliphatic compounds \\
\hline $\begin{array}{l}\text { Benzene } \\
\text { methylbenzene (toluene) }\end{array}$ & $\begin{array}{l}\text { 1,4-dihydroxy (hydroquinone) } \\
\text { 1,2,3-trihydroxy (pyrogallol) } \\
\text { 1,3,5-trihydroxy (phloroglucinol) }\end{array}$ & $\begin{array}{l}\text { caproic } \\
\text { citric } \\
\text { crotonic }\end{array}$ \\
\hline Benzoate & 3-hydroxy methyl- (m-cresol) & formic \\
\hline 2-hydroxybenzoate (salicylate) & 4-hydroxy methyl- (p-cresol) & fumaric \\
\hline 3-hydroxy & 2-chlorophenol & glutaric \\
\hline 4-hydroxy & 3-chloro & glyoxalic \\
\hline $\begin{array}{l}\text { 2,4-dihydroxy ( } \beta \text {-resorcylate) } \\
\text { 2,5-dihydroxy (gentisate) }\end{array}$ & $\begin{array}{l}\text { 4-chloro } \\
\text { 2,4-dichloro }\end{array}$ & $\begin{array}{l}\text { lactic } \\
\text { maleic }\end{array}$ \\
\hline $\begin{array}{l}\text { 2,6-dihydroxy ( } \gamma \text {-resorcylate) } \\
\text { 3,4-dihydroxy (protocatechuate) }\end{array}$ & $\begin{array}{l}\text { 3,4-dichloro } \\
\text { 3,5-dichloro }\end{array}$ & $\begin{array}{l}\text { palmitic (sodium salt) } \\
\text { propionic }\end{array}$ \\
\hline $\begin{array}{l}\text { 3,5-dihydroxy ( } \alpha \text {-resorcylate) } \\
\text { 2,3,4-trihydroxy }\end{array}$ & $\begin{array}{l}\text { pentachloro- } \\
\text { 2-amino }\end{array}$ & $\begin{array}{l}\text { 3-hydroxypropanoic } \\
\text { sorbic }\end{array}$ \\
\hline 2,4,6-trihydroxy & 2-methoxy & stearic (sodium salt) \\
\hline 3,4,5-trihydroxy (gallate) & 3-methoxy & succinic \\
\hline 3-chloro & 4-methoxy & $\mathrm{n} \& \mathrm{i}$-valeric \\
\hline 4-chloro & 2,6-dimethoxy & \\
\hline 3-chloro,4-hydroxy & 2-nitro & Aldehydes \\
\hline 3,5-dichloro & 3-nitro & acetaldehyde \\
\hline 2-bromo & 4-nitro & butyraldehyde \\
\hline 3-bromo & & crotonaldehyde \\
\hline 4-bromo & phenylacetate & formaldehyde \\
\hline 2-iodo & phenylpropenoate (cinnamate) & propionaldehyde \\
\hline 3-iodo & phenylpropionate (hydrocinnamate) & \\
\hline 4-iodo & 3-methoxy-4-hydroxy cinnamate (ferulate) & Alcohols \\
\hline 2-amino (anthranilate) & 4-hydroxyphenylalanine (tyrosine) & n \& i-butanol \\
\hline 3-amino & & $1,2 \& 2,3$-butanediol \\
\hline 4-amino & Benzyl alcohol & 3-methylbutanol \\
\hline 2-methyl (o-toluate) & 4-hydroxy benzyl alcohol & ethylene glycol \\
\hline 3-methyl (m-toluate) & & di, tri \& polyethylene glycol \\
\hline 4-methyl (p-toluate) & Heterocyclic aromatic compounds & ethanol \& 2-methoxyethanol \\
\hline 2-methoxy & & glycerol \\
\hline 3-methoxy & pyridine & methanol \\
\hline 4-methoxy (p-anisate) & 3-pyridine carboxylate (nicotinate) & octanol \\
\hline 3,4,5-trimethoxy & 2,6-pyridinedicarboxylate (dipicolinate) & pentanol \\
\hline 4-hydroxy-3- methoxy (vanillate) & purine & propanol \& 1-amino-2-propanol \\
\hline 4-hydroxy-3,5-dimethoxy (syringate) & adenine & 1,2-propanediol \\
\hline 2-nitro & xanthine & \\
\hline 2-acetyl (acetylsalicylate) & $\begin{array}{l}\text { indole } \\
\text { tryptophane }\end{array}$ & $\begin{array}{l}\text { Amines } \\
\text { butylamine }\end{array}$ \\
\hline Benzaldehyde & uracil & trimethylamine \\
\hline 4-hydroxy-3,5-dimethoxy (syringaldehyde) & quinoline & triethanolamine \\
\hline 4-hydroxy-3-methoxy (vanilline) & 2-furaldehyde (furfural) & \\
\hline $\begin{array}{l}\text { o, m, p-dicarboxybenzene (o, m, p-phthalate }) \\
\text { dimethyl o-phthalate \& p-phthalate } \\
\text { diethyl o-phthalate }\end{array}$ & Aliphatic compounds & $\begin{array}{l}\text { Ketones } \\
\text { acetone } \\
\text { methyl ethyl ketone }\end{array}$ \\
\hline di-n-butyl o-phthalate & Hydrogen cyanide & Esters \\
\hline Butylbenzyl o-phthalate & & ethyl, methyl \& vinyl acetate \\
\hline Nitrobenzene & $\begin{array}{l}\text { Acids } \\
\text { acetic }\end{array}$ & $\begin{array}{l}\text { butyl, ethyl \& methyl acrylate } \\
\text { methyl butyrate }\end{array}$ \\
\hline 3-nitrobenzene sulfonate & $\begin{array}{l}\text { acrylic } \\
\text { adipic }\end{array}$ & methyl propionate \\
\hline Hydroxybenzene (phenol) & 4-aminoadipic & Ethers \\
\hline 1,2-dihydroxybenzene (catechol) & n- \& i-butyric & ethylene glycol monoethyl ether \\
\hline 1,3-dihydroxy (resorcinol) & 3-hydroxybutyric & methyl butyl ether \\
\hline
\end{tabular}


During the same period, only 2 UASB reactors (12.5\% of the reactors) were built when this system was already the anaerobic leading technology in other industrial sectors. During the following years, UASB systems progressed (34.8\% of the reactors), but globally reactors with static packing (UAF, DSFF, hybrid) remained majority ( $43.5 \%$ of the reactors). This situation does not appear to be related with sludge granulation difficulties. During the last 8 years, 1 Fluidised bed and 5 EGSB reactors have been also applied. Their small number is probably due to the fact that these technologies have reached commercialisation only recently.

Contrarily to what happens in the other industrial sectors, for chemistry and petrochemistry, all the types of high rate reactors are not interchangeable. Again, a precise example corresponds to the case of the effluents generated during the production of terephthalic acid. Whereas full-scale DSFF and hybrid reactors are usually able to remove efficiently TA, besides benzoic acid and acetic acid (the two other main organic pollutants present in this type of wastewater), single stage anaerobic contact and UASB reactors are often unable to achieve it, or at least much less efficiently and after a long lag phase (Vanduffel, 1993; Pereboom et al., 1994; Young et al., 2000). TA representing 7 to $50 \%$ of this wastewater COD, the performances of this last class of reactors may be limited to 50-60\% COD removal (Table 2, reactors 19, 39) compared to a minimum of $75-80 \%$ for the others (e.g. reactors $14,17,18,22,32$ in Table 2). Such difference in behaviour is probably related to an improved retention of biomass. Actually, TA primary degraders are characterised by an extremely low growth rate, which indicates that they should be retained more easily in reactors with some kind of packing as was observed by Kleerebezem et al. (1999b) in a study with UASB and hybrid reactors fed with TA as sole carbon and energy source. This explanation however is insufficient due to the fact that acetic and benzoic acids have been shown to inhibit the methanisation of TA (Fajardo et al., 1997; Kleerebezem et al, 1999a). The degradation of all the organic compounds in only one reactor thus requires the separation in space of two distinct bacterial populations. The first population has for role to eliminate the benzoic and acetic acids and by the same way, to detoxify the medium for a second population specialised in the degradation of terephthalic acid. Such a physical separation is possible only when the biomass is distributed on all the height of the digesters and these are operated in a plug flow mode which allows the formation of concentration gradients. This may be the case of DSFF reactors due to the disposition of the packing available for biomass fixation but also to hybrid reactors for which the biomass is physically separated between a sludge bed at the bottom and a bacterial film on support at the top. This advantage does not exist however in conventional single stage perfectly mixed anaerobic contact and UASB reactors.

Another example of the importance of the type of reactor corresponds to the effluents containing formaldehyde. As indicated previously, this last compound is strongly toxic, it is however biodegradable below a certain concentration for which, an equilibrium between biomass growth and decay rates can be reached (Gonzalez-Gil et al., 1999). A simple dilution of the wastewater can thus make it possible to eliminate toxicity and by the same way to avoid an expensive chemical pre-treatment such as the one described above. Dilution with river water being prohibited, a dilution in closed loop with the water coming out of the digester is possible. The level of dilution necessary (10 to 30 to reach less than $0.5 \mathrm{~g}$ formaldehyde/L) requires however a very high rate of recirculation involving high water upflow velocities that only fluidised bed and EGSB reactors are able to tolerate. This scheme corresponds to the solution chosen for the effluents of the companies Caldic Europoort and DuPont, both in the Netherlands, which manufacture respectively formaldehyde and thermoplastics (Table 2, reactors 30 and 45). Such design has been shown also at lab scale as a good option for the treatment of high formaldehyde binding DMT wastewaters (Frankin et al., 1994a), and has been recently implemented at full scale in The Netherlands and Turkey (Table 2, reactors 58 and 59).

Due to the adsorptive properties of activated carbon, several lab-scale experiments (Table 3) have shown also that fluidised bed reactors packed with this type of carrier could deal with wastewaters (e.g. refinery stripper bottoms, phenolic resins, 2,4-D production) containing high concentrations of various toxicants otherwise difficult to treat with more classical anaerobic biological means (Gardner et al., 1988; Goedertz et al., 1990; Wilson et al., 1997). To date however these systems seem not to have reached practical application.

\section{CONCLUSIONS}

Although anaerobic digestion is already applied at least in 80 chemical and petrochemical companies, its development in this industrial sector remains limited until now. The capacity of growth is however very high and more interest on its application has pointed out these last 4 years. An expansion similar to that met for terephthalic acid is indeed, possible for all the effluents already treated by this way on an industrial scale 
(Table 2) and beyond for all the effluents which contain the molecules mentioned in Table 4. The still low growth rate of anaerobic digestion in this industry seems related to a lack of adequate promotion. It is surprising for instance, that while an UASB reactor is in operation since 1986 to treat the wastewaters of phenol production, no other reactor has been built to treat the same type of effluent since this date. It must be emphasised that the success of a project in this sector will be only the result of a study undertaken with rigour. Particularly the operation of a pilot scale unit on the industrial site is strongly recommended before the implementation of a full-scale unit.

\section{ACKNOWLEDGEMENTS}

This work was performed during a stay as visiting Professor at the Biotechnology Department of the Metropolitan Autonomous University, Campus Iztapalapa in Mexico City within a collaboration programme between this university and the «Institut de Recherche pour le Développement» from France. Earlier versions of this paper have been presented in 1996, in French at the «Journées Industrielles sur la digestion anaérobie» at Narbonne, France and in Spanish at the «IV Seminario Taller Latinoamericano, Tratamiento Anaerobio de Aguas Residuales» at Bucaramanga, in Colombia. Lennart Huss \& Bo Hallin (Purac), Leo Habets \& Martin Tielbaards (Paques), Robert Landine \& Ian Page (ADI), Victor Flores \& George Zoutberg (Biothane), JeanJacques Disetti (Proserpol), Lucina Equihua \& D. Marchand (Degrémont), Jeffrey Bryant (Badger), Haruki Ikemoto (Shinko Pantec), Toine Schouten (Grontmij), Denis Totzke (Applied Technologies), Marc Eeckhaut (Enviroasia), Hans Grootaerd (Biotim) and A. G. Salerno (Biotecs) are gratefully acknowledged for sending the reference list of their respective companies. Special thanks go to Alejandro Olmos Dichara for his help in the paper formatting and encouragement to publish it.

\section{REFERENCES}

Anonym (1996). Anaerobes tackle industrial effluent. Water Quality International, January/February, p. 34.

Araya P., Aroca G., Chamy R. (1999). Anaerobic treatment of effluents from an industrial polymers synthesis plant. Waste Management, 19, 141-146.

Bekker de P., Jans T. and Piscaer P. (1983). Anaerobic treatment of formaldehyde containing waste water. In: Proc. of the European Symposium AWWT, W. J. van den Brink (ed.), 23-25 November 1983, Noordwijkerhout, TNO Corporate Communication Department, The Hague, the Netherlands. pp. 449-463.

Blum D. J. W. and Speece R. E. (1991). A database of chemical toxicity to environmental bacteria and its use in interspecies comparisons and correlations. Res.J. WPCF, 63, 198-207.

Borghans A. J. M. L. and van Driel A. (1988). Application of the biothane ${ }^{\circledR}$ UASB reactor to a chemical waste water, containing phenol and formaldehyde. In: Poster-Papers, 5th Int. Symp. Anaerobic Digestion, Bologna, Italy, A. Tilche and A. Rozzi (eds.), Monduzzi Editore, Bologna, Italy.pp. 627-630.

Boulenger P., Driessen W., van de Werfhorst, Tielbaard M. (2000). Anaerobic effluent treatment by a pilot and full scale plant at a chemical industrial complex. Wat. Sci. Tech. 42(5-6), 283-287 (this issue).

Brookes P. R. and Livingston A. G. (1994). Biotreatment of a point-source industrial wastewater arising in 3,4-dichloroaniline manufacture using an extractive membrane bioreactor. Biotechnol. Prog., 10, 65-75.

Chou W. L., Speece R. E. and Siddiqi R. H. (1978). Acclimation and degradation of petrochemical wastewater components by methane fermentation. Biotech. Bioeng. Symp., 8, 391-414.

Constable S. W. C., Kras R. (1998). Selection, start-up and operation of an anaerobic pretreatment system for wastewater from a thermoplastic production facility. In: Proc. 71st Annual Water Environment Federation Conf., 3-7 October 1998, Orlando, Florida, USA, 10 pages

Dangcong P., Xingwen Z., Qiting J., Liangkui X. and De Z. (1994). Effects of seed sludge on the performance of UASB reactors for treatment of toxic wastewater. J. Chem. Tech. Biotechnol., 60, 171-176.

Dohányos M, Zábrabska J. and Grau P. (1988). Anaerobic breakdown of acrylic acid. In: Proc. 5th Int. Symp. Anaerobic Digestion. E. R. Hall and P. N. Hobson (eds.), Pergamon Press, New York, USA, pp. 287-294.

Ely S. R. and Olsen G. P. (1989). Process for treatment of wastewater. US Patent. 4,826,600 (assigned to Amoco Co). 10 pages

Fajardo C., Guyot J. P., Macarie H. and Monroy O. (1997). Inhibition of anaerobic digestion by terephthalic acid and by products. Wat. Sci. Tech., 36(6-7), 83-90.

Fdz-Polanco, Hidalgo M. D., Fdz-Polanco M. and García Encina P. A. (1999). Anaerobic treatment of polyethylene terephthalate (PET) wastewater from lab to full scale. Wat. Sci. Tech., 40(8), 229-236.

Ferguson J. F., Brian J. E. and Benjamin M. M. (1984). Neutralization in anaerobic treatment of an acidic waste. Wat. Res., 18, 573-580.

Ferrel N. J., Young J. C. (1993). Biological treatment of natural gas plant wastewater. In: Proc. 48th Purdue Ind. Wast. Conf., Lewis Publishers, Chelsea, Michigan, USA, pp. 455-468.

Fueillette C. (1996). Inventory of the industrial anaerobic reactors in France. In: Mémoire des Journées Industrielles sur la Digestion Anaérobie, 17-19 June 1996, Narbonne, France, INRA, pp. 1-9 (in French). 
Frankin R. J., Koevoets W. M. A., Versprille A. I. (1994a). Application of the Biobed ${ }^{\circledR}$ system for formaldehyde containing dimethylterephthalate (DMT) waste water. In: Poster-Papers, 7th Int. Symp. Anaerobic Digestion. 23-27 January 1994, Cape Town, South Africa. pp. 244-247.

Frankin R. J., van Gils W. M. A., Wermeling, R. J. P. (1994b). Full scale anaerobic treatment of Shell wastewater containing benzoate with the Biothane ${ }^{\circledR}$ UASB process. In: Poster-Papers, 7th Int. Symp. Anaerobic Digestion. 23-27 January 1994, Cape Town, South Africa. pp. 248-251.

Gardner D. A., Suidan M. T. and Kobayashi, A. (1988). Role of GAC activity and particle size during the fluidized-bed anaerobic treatment of refinery sour water stripper bottoms. J. WPCF, 60, 505-513.

Goedertz J. G., Weber S. and Ying W. (1990). Startup and operation of an activated carbon (AnBAC) process for treatment of a high strengh multicomponent inhibitory wastewater. Environ. Progress, 9, 110-117.

Gonzalez-Gil G., Kleerebezem R., van Aelst A., Zoutberg G. R., Versprille A. I. and Lettinga G. (1999). Toxicity effects of formaldehyde on methanol degrading sludge and its anaerobic conversion in Biobed ${ }^{\circledR}$ expanded granular sludge bed (EGSB) reactors. Wat. Sci. Tech., 40(8), 195-202.

Harvey L. M. and Rubiano J. C. (1983). The anaerobic approach: a better answer. Ind. Waste, 29, 22-25.

Henry M. and Varaldo C. (1988). Anaerobic digestion treatment of chemical industry waste-waters at the Cuise-Lamotte (Oise) plant of Société française Hoechst. In: Proc. 5th Int. Symp. Anaerobic Digestion. E. R. Hall and P. N. Hobson (eds.), Pergamon Press, New York, USA, pp. 479-486.

Hirata Y. S. (1994). Experiences and perspectives of industrial wastewater anaerobic treatment in Brazil. In: Proc. III Taller y Seminario Latinoamericano Tratamiento Anaerobio de Aguas Residuales. M. Viñas, M. Soubes, L. Borzaconi, L. Muxi (eds.), 25-28 October 1994, Montevideo, Uruguay, pp. 281-291 (In Portuguese).

Hovious J., Conway R. and Ganze C. (1973). Anaerobic lagoon pretreatment of petrochemical wastes. J. WPCF, 45, 71-84.

Kleerebezem R. (1999). Anaerobic Treatment of Phthalates: Microbiological and Technological Aspects. PhD thesis, University of Wageningen, The Netherlands (ISBN 90-5808-127-3).

Kleerebezem R., Hulshoff Pol L. W. and Lettinga G. (1999a). The role of benzoate in anaerobic degradation of terephthalate. Appl. Environ. Microbiol., 65, 1161-1167.

Kleerebezem R., Ivalo M., Hulshoff Pol L. W. and Lettinga G. (1999b). High rate treatment of terephthalate in anaerobic hybrid reactors. Biotechnol. Prog., 15, 347-357.

Koyama O., Kamagata Y. and Nakamura K. (1994). Degradation of chlorinated aromatics by Fenton oxidation and methanogenic digester sludge. Wat. Res., 28, 895-899.

Macarie H. (1992). Anaerobic Treatment of the wastewater of a petrochemical plant producing an aromatic compound, terephthalic acid (1,4-benzenedicarboxylic acid). PhD thesis, Université de Provence, France.

Macarie H., Noyola A. and Guyot J. P. (1992). Anaerobic treatment of a petrochemical wastewater from a terephthalic acid plant. Wat. Sci. Tech., 25(7), 223-235.

Marx F. T. I. (1994). Mossgas reaction water treatment plant. In: Poster-Papers, 7th Int. Symp. Anaerobic Digestion. 23-27 January 1994, Cape Town, South Africa, pp. 279-282.

Monroy O., Famá G., Meraz M., Montoya L. and Macarie H. (2000). Anaerobic digestion for wastewater treatment in Mexico: state of the technology. Wat. Res., 34, 1803-1816.

Nemer R., Zaloum R., Gehr R. and Guiot S. R. (1994). Evaluation of available techniques for assessing anaerobic treatability of chemical effluents. In: Proc. 17ème Symp. Int. sur le Traitement des Eaux Usées. 15-17 November 1994, Montréal,, Collection Environnement, Université de Montréal, vol. 2, pp. 32-45.

Noyola A., Macarie H., Varela F., Landrieu S., Marcelo R., Rojas M. A. (2000). Upgrade of a petrochemical wastewater treatment plant by an upflow anaerobic pond. Wat. Sci. Tech., 42(5-6), 269-276 (this issue).

Page I. C., Cocci A. A., Grant S. R., Wilson D. R., Landine R. C. (1999). Single stage anaerobic hybrid treatment of a polyesterintermediate production wastewater. In: Proc. Int. Conf. Waste Minimisation \& End of Pipe Treatment in Chemical and Petrochemical Industries, 14-18 November, 1999, Merida, Yucatan, Mexico, pp. 529-532.

Page I. C., Wilson D. R., Cocci A. A., Landine R. C. (1998). Anaerobic hybrid treatment of terephthalic acid wastewater. In: Proc. 71st Annual Water Environment Federation Conf., 3-7 October 1998, Orlando, Florida, USA, vol 3, pp. $575-586$.

Pereboom J. H. F., De Man G. and Su I. T. (1994). Start-up of full scale UASB reactor for the treatment of terephthalic acid wastewater. In: Poster Papers, 7th Int. Symp. Anaerobic Digestion, 23-27 January 1994, Cape Town, South Africa, pp. 307312.

Pulgarin C. Adler N., Péringer P. and Comninellis C. (1994). Electrochemical detoxification of a 1,4-benzoquinone solution in wastewater treatment. Wat. Res., 28, 887-893.

Roy M. A. and Durand M. B. (1994) The methanisation at French Company Hoechst. In: Journées Techniques Méthanisation des Effluents Industriels et Valorisation Energétique, 10 Ans d'Expériences. 11 October 1994, Compiègne, ATEE, France, 7 pages (in French).

Seyfried C. F. and Austermann-Haun U. (1997). Full scale experiences with the anaerobic pre-treatment of industrial wastewater in Germany. In: Proc. 8th Int. Conf. Anaerobic Digestion, 25-29 May 1997, Sendai, Japan, vol 1, pp. 75-82.

Shelley S. (1991). The company's innovative biodegradation technique-using anaerobic bacteria to degrade chemical waste-wins the 1991 Kirkpatrick chemical engineering achievement award. Chem. Eng., 98(12), 90-93. 
Szewzyk U. and Schink B. (1989). Methanogenic degradation of hydroquinone in an anaerobic fixed-bed reactor. Appl. Microbiol. Biotechnol., 32, 346-349.

Todini O. and Hulshoff Pol L. (1992). Anaerobic degradation of benzaldehyde in methanogenic granular sludge: the influence of additional substrates. Appl. Microbiol. Biotechnol., 38, 417-420.

Totzke D. E. (1999). 1999 anaerobic treatment technology overview. In: Course on Anaerobic Treatment of High Strength Agricultural and Industrial Waste, 20-21 September 1999, University of Wisconsin, Milwaukee, WI, USA, 14 pages.

Vanduffel J. M. (1993). Anaerobic treatment of organic acids. National Conference on Anaerobic Treatment of Complex Wastewaters, Breda, The Netherlands (in Dutch).

Wang Y. T. (1990). Methanogenic degradation of ozonation products of biorefractory or toxic aromatic compounds. Wat. Res., 24, $185-190$.

Wilson G. J., Suidan M. T., Maloney S. W., Brenner R. C. (1997). The biodegradation of 2,4-D industrial wastewater utilising a pilot-scale anaerobic GAC-FBR in Eastern Europe. In: Proc. 70th Annual Water Environment Federation Conf., 18-22 October 1997, Chicago, Illinois, USA, 9 pages.

Wirtz R. A. and Dague R. R. (1993). Anaerobic treatment of a waste furfural production wastewater. Waste Management, 13, 309315.

Yi. Q., Yibo W. and Huiming Z. (1994). Efficacy of pretreatment methods in the activated sludge removal of refractory compounds in coke-plant wastewater. Wat. Res., 28, 701-707.

Young J. C. (1991). Factors affecting the design and performance of upflow anaerobic filters. Wat. Sci. Tech., 24(8), 133-155.

Young J. C., Kim I. S., Page I. C., Wilson D. R., Brown G. J. and Cocci A. A. (2000). Two stage anaerobic treatment of purified terephthalic acid production wastewaters. Wat. Sci. Tech., 42(5-6), 277-282 (this issue).

Young J. C. and Young H. W. (1991). Full scale treatment of chemical process wastes using anaerobic filters. Res. J. WPCF, 63, $153-159$.

Zoutberg G. R. and de Been P. (1997). The Biobed ${ }^{\circledR}$ EGSB (expanded granular sludge bed) system covers shortcomings of the upflow anaerobic sludge blanket reactor in the chemical industry. Wat. Sci. Tech., 35(10), 183-188. 